\title{
A quick and qualitative assessment of gross motor development in preschool children
}

Kakebeeke, Tanja H ; Chaouch, Aziz ; Knaier, Elisa ; Caflisch, Jon ; Rousson, Valentin ; Largo, Remo H ; Jenni, Oskar G

\begin{abstract}
There is a need for a quick, qualitative, reliable, and easy tool to assess gross motor development for practitioners. The aim of this cross-sectional study is to present the Zurich Neuromotor Assessment-Q (ZNA-Q), which assesses static and dynamic balance in children between 3 and 6 years of age in less than 5 min. A total of 216 children (103 boys; 113 girls; median age 4 years, 4 months; interquartile range 1 year, 3 months) were enrolled from day-care centers, kindergartens, and schools, and were tested with 5 different gross motor tasks: standing on one leg, tandem stance, hopping on one leg, walking on a straight line, and jumping sideways. All ordinal measures (consisting of qualitative measures and scales) featured a marked developmental trend and substantial inter-individual variability. Test-retest reliability was assessed on 37 children. It varied from .17 for tandem stance to .43 for jumping sideways for the individual tasks, and it was .41 and .67 for the static and dynamic balance components, respectively. For the whole ZNA-Q, test-retest reliability was .7.Conclusion: Ordinal scales enable practitioners to gather data on children's gross motor development in a fast and uncomplicated way. It offers the practitioner with an instrument for the exploration of the current developmental motor status of the child. What is Known: - Measurement of gross motor skills in the transitional period between motor mile stones and quantitative assessments is difficult. - Assessment of gross motor skills is relatively easy. What is New: - Supplementary and quick gross motor test battery for children for practitioners. • Normative values of five gross motor skills measured with ordinal scales.
\end{abstract}

DOI: https://doi.org/10.1007/s00431-019-03327-6

Posted at the Zurich Open Repository and Archive, University of Zurich ZORA URL: https://doi.org/10.5167/uzh-181900

Journal Article

Accepted Version

Originally published at:

Kakebeeke, Tanja H; Chaouch, Aziz; Knaier, Elisa; Caflisch, Jon; Rousson, Valentin; Largo, Remo H; Jenni, Oskar G (2019). A quick and qualitative assessment of gross motor development in preschool children. European Journal of Pediatrics, 178(4):565-573.

DOI: https://doi.org/10.1007/s00431-019-03327-6 


\section{A quick and qualitative assessment of gross motor development in preschool children}

T. H. Kakebeeke ${ }^{1,2, *}$, A. Chaouch ${ }^{4}$, E. Knaier ${ }^{1}$, J. Caflisch ${ }^{1,2}$, V. Rousson ${ }^{4}$, R. H. Largo ${ }^{1}$, O.

G. Jenni ${ }^{1,2,3}$

tanja.kakebeeke@kispi.uzh.ch;

aziz.chaouch@chuv.ch;

elisa.knaier@kispi.uzh.ch;

jon.caflisch@kispi.uzh.ch;

valentin.rousson@chuv.ch;

brlargo@bluewin.ch;

oskar.jenni@kispi.uzh.ch

* Address correspondence to:

Tanja H. Kakebeeke

Child Development Center

University Children's Hospital Zürich

Steinwiesstrasse 75

CH-8032 Zürich

Switzerland

e-mail: (tanja.kakebeeke@kispi.uzh.ch)

fax: ++ 41442667164

Footnote

${ }^{1}$ Child Development Center, University Children's Hospital Zürich, Zürich, Switzerland

${ }^{2}$ Giedion Risch Foundation, Zürich, Switzerland

${ }^{3}$ Children's Research Center, University Children's Hospital Zürich, Zürich, Switzerland

${ }^{4}$ Division of Biostatistics, Institute of Social and Preventive Medicine, University Hospital,

Lausanne, Switzerland 


\section{Click here to access/download \\ Electronic Supplementary Material Stat_appendix_EJPE-D-18-00929.docx}




\section{Abstract}

There is a need for a quick, qualitative, reliable and easy tool to assess gross motor development for practitioners. The aim of this cross-sectional study is to present the Zurich Neuromotor Assessment-Q (ZNA-Q), which assesses static and dynamic balance in children between 3 and 6 years of age in less than 5 minutes. A total of 216 children (103 boys; 113 girls; median age: 4 years 4 months, interquartile range: 1 year 3 months) were enrolled from daycare centres, kindergartens, and schools, and were tested with 5 different gross motor tasks: standing on one leg, tandem stance, hopping on one leg, walking on a straight line, and jumping sideways. All ordinal measures (consisting of qualitative measures and scales) featured a marked developmental trend and substantial interindividual variability. Test-retest reliability was assessed on 37 children. It varied from $\theta . .19 .17$ for tandem stance to $\theta .5043$ for hopping on en for the individual tasks, and it was $\theta . .41$ and $\theta . .67$ for the static and dynamic balance components, respectively. For the whole ZNA-Q, test-retest reliability was $\theta$. 70.

Conclusion: Ordinal scales enable practitioners to gather data on children's gross motor development in a fast and uncomplicated way. It offers the practitioner with an instrument for the exploration of the current developmental motor status of the child.

\section{Abbreviations:}

CAMs: contralateral associated movements

DB: dynamic balance

FM: fine motor

PM: pure motor

PMDA: Poor Man's Data Augmentation

SDS: standard deviation score

SB: static balance

ZNA: Zurich Neuromotor Assessment

ZNA-2: Zurich Neuromotor Assessment second edition

\section{Keywords}

Zurich Neuromotor Assessment, quick version, gross motor skills

\section{What is Known:}

- Measurement of gross motor skills in the transitional period between motor mile stones and quantitative assessments is difficult

- Assessment of gross motor skills is relatively easy

\section{What is New:}

- Supplementary and quick gross motor test battery for children for practitioners

- Normative values of five gross motor skills measured with ordinal scales 


\section{Introduction}

In 2018, an updated version of the ZNA, the ZNA-2 [13], was published with new normative data for children's motor proficiency from 3 to 18 years. The ZNA-2 presents the developmental course and inter-individual variation of timed performance and quality of movements in a set of motor tasks of variable complexity for children between 3 and 18 years $[13,16,17]$

The greatest challenge for the ZNA-2 was to integrate data from children who were not able to perform the tasks because they were too young, too poorly performed, or both. Such missing data were integrated into the estimation of the outcome model with the poor man's data augmentation (PMDA) algorithm [26]. This method enabled the incorporation of those children who could not perform a task due to their young age.

However, sometimes it occurs that an easier or similar version of the same task can be performed. For instance, a three year old may not be able to stand on either leg for more than 2 seconds but can stand still for more than 2 seconds with one foot ahead of the other on a line. This task, called tandem stance [11], can provide information about ability in static balance when standing on one leg is not possible. Likewise, information about dynamic balance can be obtained by asking a child to walk forward on a straight line when the child is not able to perform a standing long jump. For this reason, some easier items for gross motor skills may help to evaluate these skills more precisely in the younger age group and thus gain an overview of the motor developmental status of the child.

In 2012, we presented a way of testing seven basic gross motor skills in preschool children with the purpose of bridging the gap between the measurement of motor milestones for toddlers and quantifying motor behaviour in a competitive way [14]. Motor performance was quantified on an ordinal scale with 5 categories. Improvements over age were presented for walking on a beam, running, taking stairs, standing on one leg, hopping on one leg, rising, and jumping down. This investigation showed that developmental trends differed considerably over the seven tasks. For instance, hopping on one leg exhibited a highly significant trend over age, whereas for running, changes over age were non-significant. Therefore, downscaling gross motor tasks precisely required that some new items were added while those without a significant developmental trend were omitted. The revised version of this easier test for gross motor skills using ordinal scales was labelled ZNA-Q.

The ZNA-Q might provide a substantial advantage for practitioners (paediatric clinicians, physiotherapist and sports teachers) who seek a quick overview of the developmental status of a child. The data from this 5 minute test allow a rough estimate of the child's gross motor development. For instance, a three-year-old child who is able to stand still one foot ahead of the other for more than 10 seconds and is able to hop on one leg more than 5 times does not immediately need a complete neuromotor assessment, which depending on the tool takes about 30 to 45 minutes $[6,9,10,13]$. 
In this study a limited set of ordinal measures for gross motor development in children below six years of age is explored. In using this tool in the future, we hope to detect quickly and easily children with large gross motor delays in the transitional period from the assessment of motor milestones to quantitative measurement of gross motor skills.

\section{Materials and method}

\section{Participants}

A total of 216 children (103 boys; 113 girls) between the ages of 3 and 6 years (median age: 4 years 4 months, interquartile range: 1 year 3 months) participated in this cross-sectional study. Additionally, 37 children participated in the study that investigated the test-retest reliability of the tool. All children were enrolled from daycare centres and kindergartens in the greater Zurich area. Children with evident medical or behavioural conditions (e.g. physically/mentally challenged) were excluded from the analysis. All the children lived in the Zurich area and together constitute a representative sample of the general local population. Special attention was given to sampling from districts with low, medium, and high socioeconomic backgrounds. The study was approved by the institutional review board of the Canton of Zurich (KEK-ZH-Nr. StV-40/07) and performed according to the Declaration of Helsinki. All families received a study description and provided written informed consent.

\section{Measurements}

The original ZNA is a standardized procedure that was specifically designed to describe neuromotor development in typically developing children from 5 years to 18 years of age. It focuses on variability and age changes in motor proficiency $[13,16-18]$ by measuring the speed of motor tasks and the quality of movements (i.e., the intensity of contralateral associated movements: CAMs). Motor proficiency is measured on five components: fine motor adaptive tasks (FM-motor tasks with visuo-spatial perception), pure motor tasks (PM-motor tasks with as little visuo-perceptual challenges as possible), dynamic balance (DB), static balance (SB), and movement quality (CAMs). The assessment of motor proficiency was later extended to children between 3 and 5 years of age using a customized version of the test (10).

In the updated version of the ZNA, the ZNA-2, we use essentially the same items and components as in the original [18]. To take account of improvements with age in performance, the number of repetitions was increased for easier comparison of younger with older children. However, the task was sometimes too difficult for the younger age group. The FM and PM tasks could not be made easier, and we had sufficient data from our total 9 tasks $(3 \times \mathrm{FM}$ and $6 \times \mathrm{PM})$ 

added to the component of SB with the aim of providing a better estimation of the static balance competencies of a child. For DB, 3 ordinal scales with 5 levels ranging from 0 (best possible performance) to 4 (worse possible performance) were added to provide us with more information on poorly performing children. For consistency, performance on static balance was also expressed on an ordinal scale with 5 levels. This enabled us to describe the gross motor development on 5 different tasks and grouped these 5 items under the name ZNA-Q. All ZNA-Q items, the descriptions of the ordinal scales and pictures of children doing the tasks are presented in Table I and

Figure 1. The ZNA-Q was performed during the same session as the ZNA-2.

Insert Table I and Figure 1 here

\section{Static balance (SB)}

The child is asked to stand for as long as possible on one leg on a board marked with stripes. The stopwatch is

started as soon as one foot is lifted and stopped when the child loses balance or shifts the standing foot beyond one

stripe. The child performs the task first with the dominant (D) foot, thereafter the non-dominant (ND) foot. This routine is performed twice if the child does not reach the maximal time of 30s during the first round. If the child performs the task twice, the longer time is used for subsequent calculations. The recorded time is then converted to an ordinal score (see Figure 1).

\section{Tandem stance}

Tandem stance is a new item for the component SB. Tandem stance is a clinical measure of static balance considered to assess postural steadiness by a time measurement [11]. The child stands for as long as possible with one foot in front of the other, heel-to-toe. The stopwatch is started as soon as tandem stance is adopted and stopped when the child loses equilibrium or shifts at least one foot out of the correct position. As for standing on one leg, the recorded time is converted to an ordinal score (see Figure 1). 
For hopping on one leg the examiner stands in front of the child and demonstrates how to hop on one leg. The child has two chances to hop on each leg separately. A hop is considered successful when take-off and landing is achieved on the same foot.

\section{Walking forward on a straight line}

The examiner demonstrates to the child how to walk on a straight line of 4 meters consisting of an elastic band stretched along the floor and the child is asked to do so likewise afterwards. The child has two chances to complete the task.

\section{Jumping sideways}

The examiner demonstrates to the child how to lift off the ground with two feet parallel to the elastic band, make a touch-down and jump back. An important feature is lift off and touch down with two feet simultaneously. The child has two chances to complete the task.

\section{Procedure}

Subjects were tested in their own day-care centre or kindergarten and recorded individually on digital video. The examiners were five experienced ZNA testers who had all been trained and supervised by TK and JC. Tests always took place individually in a separate room. All tasks were performed in the same order by all children. The qualitative gross motor skills (SB and $\mathrm{DB}$ ) were always tested in the same order in relation to the quantitative items. The examiner explained verbally and demonstrated how to perform the tasks. If the child did not understand a task and did something different, a second demonstration was provided. Total test time for the 5 items was less than 5 minutes.

\section{Statistical analyses}

Ordered data from the five motor tasks was modelled as a function of age and sex using a multinomial logit regression model (see appendix for more details). Simpler models (e.g. models with partial or full proportional odds) were also investigated and the quality of their fit was compared using likelihood ratio tests. As in Kakebeeke et al. (2012), the developmental trend was visualized by plotting the expected ordinal score as a function of age and sex and raw data were then converted into standard deviation scores (SDS) (see appendix for more details). An SDS is a standardized measure of motor performance adjusted for age and sex that is approximately normally 
distributed with a zero mean and a unit variance in the normative sample. Positive values are associated with above-average performance and negative values with below-average performance. Component SDSs were calculated by summing SDSs from individual tasks and standardizing this sum to have zero mean and unit variance in the normative sample.

The test-retest reliability of individual task and component SDSs was estimated using Spearman's rank correlation coefficient. This coefficient provides a robust alternative to the intra-class correlation coefficient (ICC) [23] when the systematic error (i.e. bias due to a learning or fatigue effect) between two SDSs measured on the same child is not penalized. A 95\% confidence interval (CI) for this rank correlation was calculated using Fisher's transformation [8].

Tasks for the ZNA-Q and the ZNA-2 were performed during the same session. This enabled us to investigate the association between SDSs from the two tests at the component level. However, we note that ordinal scores for the task standing on one leg in the ZNA-Q were not obtained from a separate assessment. Rather they were obtained by applying a categorization to the time measurements observed in the same task in the ZNA-2. It follows that a naïve correlation analysis between SDSs from the two tests on the static balance component would produce biased results with enlarged correlation coefficients because the test-retest variability is not accounted for. In order to properly incorporate the test-retest variability into our correlation analysis, results from the ZNA-Q obtained at the first visit were correlated with those results from the ZNA-2 obtained at the second visit. Similarly, results from the ZNA-Q obtained at the second visit were correlated with those results of the ZNA-2 obtained at the first visit. This was performed both for the SB and DB components. Each child thus contributed two pairs of measurements in the correlation analysis and the rank correlation between SDSs of the two tests was calculating after pooling the two pairs of measurements for each child. A 95\% CI for this rank correlation was calculated using a non-parametric bootstrap approach by resampling individuals with replacement in the original dataset. This ensured a proper handling of the dependence in the data since each individual had two pairs of measurements.

\section{Results}

\section{Modelling}

Motor performance for standing on one leg, tandem stance, and hopping on one leg were adequately described by the full proportional odds model $(\mathrm{p} \geq .547 p \geq 0.547$; likelihood ratio test compared to a multinomial logit model). However, a partial proportional odds model was used for the tasks walking on a straight line and jumping sideways ( $\mathrm{p} \leq .015 p \leq 0.015$; likelihood ratio test compared to a full proportional odds model). No evidence of a significant lack of fit was observed for any of the five tasks ( $\mathrm{p} \geq .548-p \geq 0.548$ ). 


\section{Developmental course}

Panels in the top row of figure 1 illustrate the evolution of the expected ordinal score calculated on the five tasks as a function of age and sex. Lower scores indicate better performance. All tasks featured a marked developmental trend ( $\mathrm{p} \leq .001-p \leq 0.001)$. With the exception of jumping sideways, girls performed better than boys in all gross motor tasks, but the gender difference was statistically significant only for standing on one leg $\left(\mathrm{p}=\theta_{.} .002\right)$ and walking on a straight line $(\mathrm{p}=\theta . .048)$. Panels in the bottom row of figure 1 illustrate how the corresponding expected cumulative probability of obtaining a score below or equal to some value $k$, with $k \in\{0,1,2,3\}$, varies as a function of age and sex.

\section{Reliability}

Test-retest reliabilities of all tasks are provided in Table II (Appendix) and were estimated from the data on 37 children between 3 and 6 years of age. Children were tested twice with just one week in between the two tests. The same experimenter did the two assessments and the same room was used in the child care centres and Kindergartens. At the individual task level, the test-retest reliability was relatively low in this cohort, with rank correlation coefficients ranging from $\theta_{.} .17$ for tandem stance to $\theta . .43$ for jumping sideways. However, at the component level, the rank correlation coefficients reached $\theta . .41$ for SB and $\theta . .67$ for DB, with the total score of the whole test reaching $\theta . .70$ (95\% CI: $\left.\left[\theta_{.} .48 ; \theta_{.} .84\right]\right)$. Intra- and inter-tester reliability values on the items are available online in an earlier work of the same group, Table III [12].

\section{Correlation with SB and DB from the ZNA-2}

The rank correlation between the composite scores for SB of the ZNA-Q and the ZNA-2 was estimated at $\theta_{.} .48$ while the rank correlation for DB between ZNA-Q and ZNA-2 was $\theta . .50$. . The rank correlation for the SB and DB scores together between the two tests was $\theta . .61$. Due to the limited sample size, the uncertainty associated with these estimates was large, with the lower bound of the $95 \% \mathrm{CI}$ being $\theta_{.} .03,0 . .20$ and $\theta_{.} .24$ for SB, DB and the total score, respectively.

\section{DISCUSSION}

Developmental trends for 5 gross motor tasks of typically developing children are reported in this study. These 5 tasks form the core of the ZNA-Q, a tool which enables a rough estimate of gross motor development in children between 3 and 6 years in a time of only 5 minutes. No similarly quick tool is currently used in paediatric practice, and we hope that this simplified test allows over- and underachievers to be identified more easily. 
It is known that children undergo huge developmental leaps in their motor development between being a toddler and becoming a child. Below 2 years, motor development is described predominantly with motor milestones $[4,5,21]$, which can only inform us about the first occurrence of certain tasks. Two commonly used assessment tools provide meaningful measurements from age three $[10,12]$. However, these measurements take at least half an hour to complete and are therefore not practical for screening purposes. Other instruments cannot be used as they do not include children of age three $[6,9,15]$. An investigation into the construct of motor competence in early childhood used Rasch modelling as a test for motor proficiency in 4- to 6-year old children [27]. This modelling can also be used to assess 3-year olds [25]. However, even the short form consists of 12 items comprising fine motor tasks and tasks with balls and therefore taking too long. As we were aiming to make a quick developmental test on gross motor development for 3-6 year olds, we could not use any of the existing tests.

With the ZNA-Q, we focus on the development of gross motor abilities, described by Burton and Miller [7] as general traits or capacities of an individual, as the basis of a variety of motor skills. Of these general traits, we are interested in gross locomotor skills used in a range of movements to transport the body from one location to another including standing [24]. While fine motor performance is also important for the global evaluation of motor abilities in infants [22], the assessment of fine motor performance is less complicated and easily testable with other tools $[10,13]$. As we wanted to build a pre-screening tool for the quick assessment of motor performance, we chose to focus on gross motor abilities in the ZNA-Q. Our interest in the general traits of infants' gross motor development led us to apply the holistic model of movement competence proposed by Rudd et al. [22]. According to this model, locomotor, object control, and body coordination develop as intrinsic aspects of movement competence. However, several articles have reported that cultural background [2,3] and sex [17,20] have an impact on motor proficiency. In this article, we explore firstly the general traits of the child, independent of environment or sex, and secondly, the locomotor part (going from A to B) of motor development. Testing children below age six who are not yet competitive and are not very competent on tasks in which speed and distance are measured requires a tool such as the ZNA-Q to bridge this gap between toddler and child. This implies that assessments which apply a productoriented approach cannot be used; a process-oriented approach is likely to be more informative [19].

Earlier work by the same group on this topic [14] included some items that are no longer present in the ZNAQ. The items rising, running, and jumping down do not distinguish sufficiently between children due to too little variability after age three, and for this reason are not suitable for screening at this developmental age. Conversely, some other items were added as easier versions of tasks in the ZNA-2. Yet, because many preschool children, especially the 3-year olds, are unable to perform some tasks (especially on dynamic balance (DB)), the use of 
ordinal scales allows the inability to perform to be treated simply as the lowest category of motor performance without relying on more complex approaches such as that used in the ZNA-2 with the PMDA algorithm [23].

The test-retest reliability of the total score in the ZNA-Q was estimated at $\theta_{.} .70$, which we consider acceptable given the context, for two reasons. First, as a simplified and shorter version of the ZNA-2, the ZNA-Q contains fewer tasks and thus may be expected to have a lower test-retest reliability than the complete test battery, which was estimated at $\theta . .8 \theta .$. However, one should recall that the ZNA-Q only focuses on gross motor skills while the ZNA-2 also includes fine and pure motor tasks and contralateral associated movements. The static balance (SB) and dynamic balance (DB) components in the ZNA-2 were the least reliable dimensions, with test-retest reliabilities of $\theta_{-} 67$ and $\theta_{.} .78$ for $\mathrm{SB}$ and $\mathrm{DB}$, respectively [11]. The test-retest reliability of the ZNA-Q is thus comparable to that observed for the corresponding dimensions of the ZNA-2. Secondly and more importantly, the ZNA-Q only considers preschool children from 3 to 6 years old, while the ZNA-2 was developed for children and adolescents from 3 to 18 years of age. Preschool children are naturally not very stable in their behaviour [1], and such behavioural instability has a direct impact on the variability of motor performance. This is especially true for those as young as age three. As a consequence, the test-retest reliability in this population of very young children is anticipated to be lower than that measured in older children and adolescents. It should be noted that, if we exclude 3-year olds $(n=4)$ from our sample, the test-retest rank correlation increases somewhat, from $\theta . .70$ to $\theta$. .74. This effectively supports the fact that motor performance in younger children is inherently more difficult to assess than that of older children. With these considerations in mind, the test-retest reliability of the ZNA-Q appears to be broadly comparable to that of the ZNA-2, which is used in clinical practice. Moreover, we believe it is difficult to reasonably expect a better result when considering preschool children as young as 3 years of age. Nonetheless, additional investigations of the test-retest reliability of the ZNA-Q should be conducted on a larger sample, because our sample $(\mathrm{N}=37)$ was limited in size, which resulted in very large confidence intervals for rank correlations.

The correlation of $\theta . .61$ between composite scores of the ZNA-Q and ZNA-2 indicates that the two tests measure some common traits of gross motor skills but do not necessarily capture the exact same information. This may be partly explained by the tasks included in and excluded from the ZNA-Q. However, we note that while the test-retest reliability measures the agreement between results obtained with the same child at two different occasions using the same test, the correlation of $\theta_{.} .61$ between the ZNA-Q and the ZNA-2 also incorporates between-test variation. Despite this additional source of variability, the intertest reliability thus appears fairly close to the test-retest reliability, which again means that we cannot expect to achieve a much higher intertest reliability than we observed in this study. Nevertheless, although the ZNA-Q may capture slightly different information from 
the SB and DB components of the ZNA-2, the clear developmental trends observed in all tasks incorporated in the

ZNA-Q suggest that this test is applicable as a pre-screening tool for gross motor performance in preschool children $\underline{\text { to assess the current developmental status of the child in clinical practice. }}$

The ZNA-Q was established with a cohort of typically developing children. Because this cohort included few how reliably the ZNA-Q is able to identify them.

\section{Acknowledgments}

We gratefully acknowledge the support of the presidents' conference of the public schools in Zurich and the educators and teachers of the child care centres for their help with recruitment. This study was supported by the Swiss National Science Foundation, grant no. 32003B_153273.

Authors' contributions

THK: Performed data collection, coded and analysed the corresponding data and wrote the manuscript

AZ: Performed statistical analysis and modelling

EK: Performed data collection and coded the corresponding data

JC: Performed data collection and corrected the manuscript

VR: Assisted in statistical data analysis

BR: Contributed to the draft and gave advice on the assessment tools

OGJ: Corrected final draft of the manuscript

\section{Funding}

This study was supported by the Swiss National Science Foundation, grant no. 32003B_153273, the Largo and

\section{Compliance with ethical standards}

Conflict of interest The authors declare that they have no conflict of interest

Informed consent Informed consent was obtained from all individual participants included in the study. Parents 
1. Anderson, P. (2002). Assessment and development of executive function (EF) during childhood. Child Neuropsychology, 8(2), 71-82. doi:10..1076/chin.8.2.71.8724

2. Barnett, L., Hinkley, T., Okely, A. D., \& Salmon, J. (2013). Child, family and environmental correlates of children's motor skill proficiency. Journal of Science and Medicine in Sport, 16(4), 332-336. doi:10._1016/j.jsams.2012.08.011

3. Barnett, L. M., Telford, R. M., Strugnell, C., Rudd, J., Olive, L. S., \& Telford, R. D. (2018). Impact of cultural background on fundamental movement skill and its correlates. Journal of Sports Sciences, 1-8. doi:10._1080/02640414.2018.1508399

4. Bax, M., \& Gillberg, C. (2009). Development: normal/delayed/disordered. In J. Aicardi (Ed.), Diseases of the nervous system in childhood (3rd ed., pp. 891-901). London: Mac Keith Press

5. Bayley, N. (2006). Bayley Scales of Infant and Toddler Development (3nd ed.). San Antonio: Pearson

6. Bruininks, R. H., \& Bruininks, B. D. (2005). Bruininks-Oseretsky Test of Motor Proficiency - Second edition (BOT-2). Minneapolis, USA: Pearson

7. Burton, A. W., \& Miller, D. A. (1998). Movement skill assessment. Leeds, United Kingdom: Human Kinetics

8. Fisher, R. A. (1936). Statistical methods for research workers. 6th edition, revised. Edinburgh: Oliver and Boyd

9. Folio, M., \& Fewell, R. (2000). Peabody Developmental Motor Scales-2. Austin, USA: PRO-ED

10.. Henderson, S. E., Sugden, D. A., Barnett, A. L., Petermann, F., Bös, K., \& Kastner, J. (2007). Movement Assessment Battery for Children - Second Edition (Movement ABC2) - Deutschsprachige Adaptation. London, UK: Harcourt Assessment

11. Jonsson, E., Seiger, A., \& Hirschfeld, H. (2005). Postural steadiness and weight distribution during tandem stance in healthy young and elderly adults. Clinical Biomechanics (Bristol, Avon), 20(2), 202-208. doi:10. 1016/j.clinbiomech.2004.09.008

12. Kakebeeke, T. H., Caflisch, J., Chaouch, A., Rousson, V., Largo, R. H., \& Jenni, O. G. (2013). Neuromotor development in children. Part 3: motor performance in 3- to 5-yearolds. Developmental Medicine and Child Neurology, 55(3), 248-256. doi:10. $.1111 / \mathrm{dmcn} .12034$

13. Kakebeeke, T. H., Knaier, E., Chaouch, A., Caflisch, J., Rousson, V., Largo, R. H., \& Jenni, O. G. (2018). Neuromotor development in children. Part 4: new norms from 3 to 18 years. Developmental Medicine and Child Neurology. doi:10._1111/dmen.13793

14. Kakebeeke, T. H., Locatelli, I., Rousson, V., Caflisch, J., \& Jenni, O. G. (2012). Improvement in gross motor performance between 3 and 5 years of age. Perceptual and Motor Skills, 114(3), 795-806. doi:10._2466/10._13.25.PMS.114.3.795-806

15. Kiphard, E. J., \& Schilling, F. (2017). KTK - Körperkoordinationstest für Kinder Bern: Hogrefe Verlag

16. Largo, R. H., Caflisch, J. A., Hug, F., Muggli, K., Molnar, A. A., \& Molinari, L. (2001). Neuromotor development from 5 to 18 years. Part 2: associated movements. Developmental Medicine and Child Neurology, 43(7), 444-453.

17. Largo, R. H., Caflisch, J. A., Hug, F., Muggli, K., Molnar, A. A., Molinari, L., Sheehy, A., \& Gasser, S. T. (2001). Neuromotor development from 5 to 18 years. Part 1: timed performance. Developmental Medicine and Child Neurology, 43(7), 436-443.

18. Largo, R. H., Rousson, V., Caflisch, J. A., \& Jenni, O. G. (2007). Zurich Neuromotor Assessment. Zurich, Switzerland: AWE Verlag

Formatted: Italian (Italy)

Formatted: Italian (Italy) 
19. Logan, S. W., Robinson, L. E., Wilson, A. E., \& Lucas, W. A. (2012). Getting the fundamentals of movement: a meta-analysis of the effectiveness of motor skill interventions in children. Child: Care, Health and Development, 38(3), 305-315. doi:10. 1111/j.1365-2214.2011.01307.x

20._ Morris, A. M., Williams, J. M., Atwater, A. E., \& Wilmore, J. H. (1982). Age and SexDifferences in Motor-Performance of 3 through 6 Year Old Children. Research Quarterly for Exercise and Sport, 53(3), 214-221. doi:Doi 10. $.1080 / 02701367.1982 .10609342$

21. Piper, M. C., \& Darrah, J. (1994). Motor Assessment of the Developing Infant: Elsevier 22. Rudd, J., Butson, M. L., Barnett, L., Farrow, D., Berry, J., Borkoles, E., \& Polman, R. (2016). A holistic measurement model of movement competency in children. Journal of Sports Sciences, 34(5), 477-485. doi:10. 1080/02640414.2015.1061202

23. Shrout, P. E., \& Fleiss, J. L. (1979). Intraclass correlations: uses in assessing rater reliability. Psychological Bulletin, 86(2), 420-428.

24. Ulrich, D. A. (2000). Test of Gross Motor Development, 2nd Ed. Austin, USA: Pro-ED. Inc.

25. Utesch, T., Bardid, F., Huyben, F., Strauss, B., Tietjens, M., De Martelaer, K., Seghers, J., \& Lenoir, M. (2016). Using Rasch modeling to investigate the construct of motor competence in early childhood. Psychology of Sport and Exercise, 24, 179-187. doi:10. $.1016 /$ j.psychsport.2016.03.001

26. Wei, G., \& Tanner, M. (1990). A Monte Carlo Implementation of the EM Algorithm and the Poor Man's Data Augmentation Algorithms. J Am Stat Assoc, 85(411), 699704.

27. Zimmer, R., \& Volkamer, M. (1987). Motoriktest für vier- bis sechsjährige Kinder [Motor Test for four- to six-year-olds]. Weinheim: Beltz Test 
355 Table I: Items measured on ordinal scales of the Zurich Neuromotor Assessment; D = dominant leg; ND = nondominant leg.

357

\begin{tabular}{lll}
\hline Components & Tasks & 3-6 years \\
\hline Static balance & Standing on one leg (D/ND) & Time in Scales (0 to 4) \\
& Tandem stance (eyes open) & Time in Scales (0 to 4) \\
Dynamic balance & Hopping on one leg (D/ND) & Count in Scales (0 to 4) \\
& Walking on a straight line forward & Scale (0 to 4) \\
& Jumping sideways & Scale (0 to 4) \\
\hline
\end{tabular}



and rank correlations between components of the ZNA-Q and ZNA-2, with 95\% confidence intervals (brackets).

Test-retest reliability

Rank correlations

$(\mathrm{N}=37)$

with ZNA-2

\section{Static balance:}

Standing on one leg (D/ND)

$$
\begin{aligned}
& \theta . .39[\theta . .08 ; \theta . .64] \\
& \theta . .17[-\theta . .17 ; \theta . .47]
\end{aligned}
$$

Tandem stance

$$
\begin{gathered}
\theta . .41[\theta . .10 ; \theta . .65] \\
\theta . .39[-\theta . .08 ; \theta . .64] \\
\theta . .43[0 . .12 ; \theta . .66]
\end{gathered}
$$

Jumping sideways

Components:

Static Balance

$\theta . .41[\theta . .09 ; \theta . .65]$

$\theta . .48[\theta . .03 ; \theta . .68]$

Dynamic Balance

$\theta . .67[\theta . .44 ; \theta . .82]$

$\theta . .50[\theta . .20 ; \theta . .75]$

Total composite score

$\theta . .70[\theta . .48 ; \theta . .84] \quad \theta . .61[0 . .24 ; 0 . .75]$

361 
362 Figure 1. Developmental trend of the five gross motor skills tasks of the ZNA-Q. Top row: 363 expected ordinal score. Bottom row: cumulative probabilities to obtain a score below or equal 364 to $k$, with $k \in\{0,1,2,3\})$. 


\begin{abstract}
There is a need for a quick, qualitative, reliable and easy tool to assess gross motor development for practitioners. The aim of this cross-sectional study is to present the Zurich Neuromotor Assessment-Q (ZNA-Q), which assesses static and dynamic balance in children between 3 and 6 years of age in less than 5 minutes. A total of 216 children (103 boys; 113 girls; median age: 4 years 4 months, interquartile range: 1 year 3 months) were enrolled from daycare centres, kindergartens, and schools, and were tested with 5 different gross motor tasks: standing on one leg, tandem stance, hopping on one leg, walking on a straight line, and jumping sideways. All ordinal measures (consisting of qualitative measures and scales) featured a marked developmental trend and substantial interindividual variability. Test-retest reliability was assessed on 37 children. It varied from .17 for tandem stance to .43 for jumping sideways for the individual tasks, and it was .41 and .67 for the static and dynamic balance components, respectively. For the whole ZNA-Q, test-retest reliability was .7.

Conclusion: Ordinal scales enable practitioners to gather data on children's gross motor development in a fast and uncomplicated way. It offers the practitioner with an instrument for the exploration of the current developmental motor status of the child.
\end{abstract}

\title{
Abbreviations:
}

CAMs: contralateral associated movements

DB: dynamic balance

FM: fine motor

PM: pure motor

PMDA: Poor Man's Data Augmentation

SDS: standard deviation score

SB: static balance

ZNA: Zurich Neuromotor Assessment

ZNA-2: Zurich Neuromotor Assessment second edition

\section{Keywords}

Zurich Neuromotor Assessment, quick version, gross motor skills

\section{What is Known:}

- Measurement of gross motor skills in the transitional period between motor mile stones and quantitative assessments is difficult

- Assessment of gross motor skills is relatively easy

\section{What is New:}

- Supplementary and quick gross motor test battery for children for practitioners

- Normative values of five gross motor skills measured with ordinal scales 
3 In 2018, an updated version of the ZNA, the ZNA-2 [13], was published with new normative data for children's motor proficiency from 3 to 18 years. The ZNA-2 presents the developmental course and inter-individual variation of timed performance and quality of movements in a set of motor tasks of variable complexity for children between 3 and 18 years $[13,16,17]$.

The greatest challenge for the ZNA-2 was to integrate data from children who were not able to perform the tasks because they were too young, too poorly performed, or both. Such missing data were integrated into the estimation of the outcome model with the poor man's data augmentation (PMDA) algorithm [26]. This method enabled the incorporation of those children who could not perform a task due to their young age.

However, sometimes it occurs that an easier or similar version of the same task can be performed. For instance, a three year old may not be able to stand on either leg for more than 2 seconds but can stand still for more than 2 seconds with one foot ahead of the other on a line. This task, called tandem stance [11], can provide information about ability in static balance when standing on one leg is not possible. Likewise, information about dynamic balance can be obtained by asking a child to walk forward on a straight line when the child is not able to perform a standing long jump. For this reason, some easier items for gross motor skills may help to evaluate these skills more precisely in the younger age group and thus gain an overview of the motor developmental status of the child.

In 2012, we presented a way of testing seven basic gross motor skills in preschool children with the purpose of bridging the gap between the measurement of motor milestones for toddlers and quantifying motor behaviour in a competitive way [14]. Motor performance was quantified on an ordinal scale with 5 categories. Improvements over age were presented for walking on a beam, running, taking stairs, standing on one leg, hopping on one leg, rising, and jumping down. This investigation showed that developmental trends differed considerably over the seven tasks. For instance, hopping on one leg exhibited a highly significant trend over age, whereas for running, changes over age were non-significant. Therefore, downscaling gross motor tasks precisely required that some new items were added while those without a significant developmental trend were omitted. The revised version of this easier test for gross motor skills using ordinal scales was labelled ZNA-Q.

The ZNA-Q might provide a substantial advantage for practitioners (paediatric clinicians, physiotherapist and sports teachers) who seek a quick overview of the developmental status of a child. The data from this 5 minute test allow a rough estimate of the child's gross motor development. For instance, a three-year-old child who is able to stand still one foot ahead of the other for more than 10 seconds and is able to hop on one leg more than 5 times does not immediately need a complete neuromotor assessment, which depending on the tool takes about 30 to 45 minutes $[6,9,10,13]$. 
In this study a limited set of ordinal measures for gross motor development in children below six years of age is explored. In using this tool in the future, we hope to detect quickly and easily children with large gross motor delays in the transitional period from the assessment of motor milestones to quantitative measurement of gross motor skills.

\section{Materials and method}

\section{Participants}

A total of 216 children (103 boys; 113 girls) between the ages of 3 and 6 years (median age: 4 years 4 months, interquartile range: 1 year 3 months) participated in this cross-sectional study. Additionally, 37 children participated in the study that investigated the test-retest reliability of the tool. All children were enrolled from daycare centres and kindergartens in the greater Zurich area. Children with evident medical or behavioural conditions (e.g. physically/mentally challenged) were excluded from the analysis. All the children lived in the Zurich area and together constitute a representative sample of the general local population. Special attention was given to sampling from districts with low, medium, and high socioeconomic backgrounds. The study was approved by the institutional review board of the Canton of Zurich (KEK-ZH-Nr. StV-40/07) and performed according to the Declaration of Helsinki. All families received a study description and provided written informed consent.

\section{Measurements}

The original ZNA is a standardized procedure that was specifically designed to describe neuromotor development in typically developing children from 5 years to 18 years of age. It focuses on variability and age changes in motor proficiency $[13,16-18]$ by measuring the speed of motor tasks and the quality of movements (i.e., the intensity of contralateral associated movements: CAMs). Motor proficiency is measured on five components: fine motor adaptive tasks (FM-motor tasks with visuo-spatial perception), pure motor tasks (PM-motor tasks with as little visuo-perceptual challenges as possible), dynamic balance (DB), static balance (SB), and movement quality (CAMs). The assessment of motor proficiency was later extended to children between 3 and 5 years of age using a customized version of the test (10).

In the updated version of the ZNA, the ZNA-2, we use essentially the same items and components as in the original [18]. To take account of improvements with age in performance, the number of repetitions was increased for easier comparison of younger with older children. However, the task was sometimes too difficult for the younger age group. The FM and PM tasks could not be made easier, and we had sufficient data from our total 9 tasks $(3 \times \mathrm{FM}$ and $6 \times \mathrm{PM})$. 

added to the component of SB with the aim of providing a better estimation of the static balance competencies of a child. For DB, 3 ordinal scales with 5 levels ranging from 0 (best possible performance) to 4 (worse possible performance) were added to provide us with more information on poorly performing children. For consistency, performance on static balance was also expressed on an ordinal scale with 5 levels. This enabled us to describe the gross motor development on 5 different tasks and grouped these 5 items under the name ZNA-Q. All ZNA-Q items, the descriptions of the ordinal scales and pictures of children doing the tasks are presented in Table I and Figure 1. The ZNA-Q was performed during the same session as the ZNA-2.

\section{Static balance (SB)}

\section{Standing on one leg (D/ND)}

The child is asked to stand for as long as possible on one leg on a board marked with stripes. The stopwatch is started as soon as one foot is lifted and stopped when the child loses balance or shifts the standing foot beyond one stripe. The child performs the task first with the dominant (D) foot, thereafter the non-dominant (ND) foot. This routine is performed twice if the child does not reach the maximal time of 30s during the first round. If the child performs the task twice, the longer time is used for subsequent calculations. The recorded time is then converted to an ordinal score (see Figure 1).

\section{Tandem stance}

85 Tandem stance is a new item for the component SB. Tandem stance is a clinical measure of static balance considered to assess postural steadiness by a time measurement [11]. The child stands for as long as possible with one foot in front of the other, heel-to-toe. The stopwatch is started as soon as tandem stance is adopted and stopped when the child loses equilibrium or shifts at least one foot out of the correct position. As for standing on one leg, the recorded time is converted to an ordinal score (see Figure 1).

\section{Dynamic balance (DB)}


93 For hopping on one leg the examiner stands in front of the child and demonstrates how to hop on one leg. The

94 child has two chances to hop on each leg separately. A hop is considered successful when take-off and landing is

95 achieved on the same foot.

96

97

98

99

100

101

102

103

104

105

106

107

108

109

110

111

112

113

114

115

\section{Statistical analyses}

117 Ordered data from the five motor tasks was modelled as a function of age and sex using a multinomial logit 118 regression model (see appendix for more details). Simpler models (e.g. models with partial or full proportional 119 odds) were also investigated and the quality of their fit was compared using likelihood ratio tests. As in Kakebeeke 120 et al. (2012), the developmental trend was visualized by plotting the expected ordinal score as a function of age 121 and sex and raw data were then converted into standard deviation scores (SDS) (see appendix for more details).

122 An SDS is a standardized measure of motor performance adjusted for age and sex that is approximately normally

123 distributed with a zero mean and a unit variance in the normative sample. Positive values are associated with 
124 above-average performance and negative values with below-average performance. Component SDSs were

125 calculated by summing SDSs from individual tasks and standardizing this sum to have zero mean and unit variance

126 in the normative sample.

127 The test-retest reliability of individual task and component SDSs was estimated using Spearman's rank correlation

128 coefficient. This coefficient provides a robust alternative to the intra-class correlation coefficient (ICC) [23] when

129 the systematic error (i.e. bias due to a learning or fatigue effect) between two SDSs measured on the same child is

130 not penalized. A 95\% confidence interval (CI) for this rank correlation was calculated using Fisher's 131 transformation [8].

132 Tasks for the ZNA-Q and the ZNA-2 were performed during the same session. This enabled us to investigate 133 the association between SDSs from the two tests at the component level. However, we note that ordinal scores for 134 the task standing on one leg in the ZNA-Q were not obtained from a separate assessment. Rather they were obtained 135 by applying a categorization to the time measurements observed in the same task in the ZNA-2. It follows that a 136 naïve correlation analysis between SDSs from the two tests on the static balance component would produce biased 137 results with enlarged correlation coefficients because the test-retest variability is not accounted for. In order to 138 properly incorporate the test-retest variability into our correlation analysis, results from the ZNA-Q obtained at 139 the first visit were correlated with those results from the ZNA-2 obtained at the second visit. Similarly, results 140 from the ZNA-Q obtained at the second visit were correlated with those results of the ZNA-2 obtained at the first 141 visit. This was performed both for the SB and DB components. Each child thus contributed two pairs of 142 measurements in the correlation analysis and the rank correlation between SDSs of the two tests was calculating 143 after pooling the two pairs of measurements for each child. A 95\% CI for this rank correlation was calculated using 144 a non-parametric bootstrap approach by resampling individuals with replacement in the original dataset. This ensured a proper handling of the dependence in the data since each individual had two pairs of measurements.

\section{Results}

\section{Modelling}

149 Motor performance for standing on one leg, tandem stance, and hopping on one leg were adequately described by 150 the full proportional odds model ( $\mathrm{p} \geq .547$; likelihood ratio test compared to a multinomial logit model). However, 151 a partial proportional odds model was used for the tasks walking on a straight line and jumping sideways ( $\mathrm{p} \leq .015$; 152 likelihood ratio test compared to a full proportional odds model). No evidence of a significant lack of fit was 153 observed for any of the five tasks ( $\mathrm{p} \geq .548)$. 
156 Panels in the top row of figure 1 illustrate the evolution of the expected ordinal score calculated on the five tasks

157 as a function of age and sex. Lower scores indicate better performance. All tasks featured a marked developmental 158 trend $(\mathrm{p} \leq .001)$. With the exception of jumping sideways, girls performed better than boys in all gross motor tasks, 159 but the gender difference was statistically significant only for standing on one leg $(\mathrm{p}=.002)$ and walking on a straight line ( $\mathrm{p}=.048)$. Panels in the bottom row of figure 1 illustrate how the corresponding expected cumulative probability of obtaining a score below or equal to some value $k$, with $k \in\{0,1,2,3\}$, varies as a function of age and sex.

\section{Reliability}

165 Test-retest reliabilities of all tasks are provided in Table II (Appendix) and were estimated from the data on 37 children between 3 and 6 years of age. Children were tested twice with just one week in between the two tests. The same experimenter did the two assessments and the same room was used in the child care centres and Kindergartens. At the individual task level, the test-retest reliability was relatively low in this cohort, with rank correlation coefficients ranging from .17 for tandem stance to .43 for jumping sideways. However, at the component level, the rank correlation coefficients reached .41 for SB and .67 for DB, with the total score of the whole test reaching $.70(95 \%$ CI: $[.48 ; .84])$. Intra- and inter-tester reliability values on the items are available online in an earlier work of the same group, Table III [12].

\section{Correlation with SB and DB from the ZNA-2}

175 The rank correlation between the composite scores for SB of the ZNA-Q and the ZNA-2 was estimated at 48 while the rank correlation for DB between ZNA-Q and ZNA-2 was .5. The rank correlation for the SB and DB scores together between the two tests was .61. Due to the limited sample size, the uncertainty associated with these estimates was large, with the lower bound of the $95 \%$ CI being $.03, .20$ and .24 for SB, DB and the total score, respectively.

\section{DISCUSSION}

182 Developmental trends for 5 gross motor tasks of typically developing children are reported in this study. These 5 183 tasks form the core of the ZNA-Q, a tool which enables a rough estimate of gross motor development in children between 3 and 6 years in a time of only 5 minutes. No similarly quick tool is currently used in paediatric practice, and we hope that this simplified test allows over- and underachievers to be identified more easily. 
It is known that children undergo huge developmental leaps in their motor development between being a toddler and becoming a child. Below 2 years, motor development is described predominantly with motor milestones $188[4,5,21]$, which can only inform us about the first occurrence of certain tasks. Two commonly used assessment tools provide meaningful measurements from age three [10,12]. However, these measurements take at least half an hour to complete and are therefore not practical for screening purposes. Other instruments cannot be used as they do not include children of age three $[6,9,15]$. An investigation into the construct of motor competence in early childhood used Rasch modelling as a test for motor proficiency in 4- to 6-year old children [27]. This modelling can also be used to assess 3-year olds [25]. However, even the short form consists of 12 items comprising fine motor tasks and tasks with balls and therefore taking too long. As we were aiming to make a quick developmental test on gross motor development for 3-6 year olds, we could not use any of the existing tests.

With the ZNA-Q, we focus on the development of gross motor abilities, described by Burton and Miller [7] as general traits or capacities of an individual, as the basis of a variety of motor skills. Of these general traits, we are interested in gross locomotor skills used in a range of movements to transport the body from one location to another including standing [24]. While fine motor performance is also important for the global evaluation of motor abilities in infants [22], the assessment of fine motor performance is less complicated and easily testable with other tools $[10,13]$. As we wanted to build a pre-screening tool for the quick assessment of motor performance, we chose to focus on gross motor abilities in the ZNA-Q. Our interest in the general traits of infants' gross motor development led us to apply the holistic model of movement competence proposed by Rudd et al. [22]. According to this model, locomotor, object control, and body coordination develop as intrinsic aspects of movement competence. However, several articles have reported that cultural background [2,3] and sex [17,20] have an impact on motor proficiency. In this article, we explore firstly the general traits of the child, independent of environment or sex, and secondly, the locomotor part (going from A to B) of motor development. Testing children below age six who are not yet competitive and are not very competent on tasks in which speed and distance are measured requires a tool such as the ZNA-Q to bridge this gap between toddler and child. This implies that assessments which apply a productoriented approach cannot be used; a process-oriented approach is likely to be more informative [19].

Earlier work by the same group on this topic [14] included some items that are no longer present in the ZNA-

212 Q. The items rising, running, and jumping down do not distinguish sufficiently between children due to too little 213 variability after age three, and for this reason are not suitable for screening at this developmental age. Conversely, 214 some other items were added as easier versions of tasks in the ZNA-2. Yet, because many preschool children, 215 especially the 3-year olds, are unable to perform some tasks (especially on dynamic balance (DB)), the use of 
ordinal scales allows the inability to perform to be treated simply as the lowest category of motor performance without relying on more complex approaches such as that used in the ZNA-2 with the PMDA algorithm [23].

The test-retest reliability of the total score in the ZNA-Q was estimated at .70, which we consider acceptable given the context, for two reasons. First, as a simplified and shorter version of the ZNA-2, the ZNA-Q contains fewer tasks and thus may be expected to have a lower test-retest reliability than the complete test battery, which was estimated at .8. However, one should recall that the ZNA-Q only focuses on gross motor skills while the ZNA2 also includes fine and pure motor tasks and contralateral associated movements. The static balance (SB) and dynamic balance (DB) components in the ZNA-2 were the least reliable dimensions, with test-retest reliabilities of .67 and .78 for SB and DB, respectively [11]. The test-retest reliability of the ZNA-Q is thus comparable to that observed for the corresponding dimensions of the ZNA-2. Secondly and more importantly, the ZNA-Q only considers preschool children from 3 to 6 years old, while the ZNA-2 was developed for children and adolescents from 3 to 18 years of age. Preschool children are naturally not very stable in their behaviour [1], and such behavioural instability has a direct impact on the variability of motor performance. This is especially true for those as young as age three. As a consequence, the test-retest reliability in this population of very young children is anticipated to be lower than that measured in older children and adolescents. It should be noted that, if we exclude 3-year olds $(n=4)$ from our sample, the test-retest rank correlation increases somewhat, from .70 to .74 . This effectively supports the fact that motor performance in younger children is inherently more difficult to assess than that of older children. With these considerations in mind, the test-retest reliability of the ZNA-Q appears to be

234 broadly comparable to that of the ZNA-2, which is used in clinical practice. Moreover, we believe it is difficult to 235 reasonably expect a better result when considering preschool children as young as 3 years of age. Nonetheless, 236 additional investigations of the test-retest reliability of the ZNA-Q should be conducted on a larger sample, 237 because our sample $(\mathrm{N}=37)$ was limited in size, which resulted in very large confidence intervals for rank 238 correlations.

The correlation of .61 between composite scores of the ZNA-Q and ZNA-2 indicates that the two tests measure 240 some common traits of gross motor skills but do not necessarily capture the exact same information. This may be 241 partly explained by the tasks included in and excluded from the ZNA-Q. However, we note that while the test242 retest reliability measures the agreement between results obtained with the same child at two different occasions 243 using the same test, the correlation of .61 between the ZNA-Q and the ZNA-2 also incorporates between-test 244 variation. Despite this additional source of variability, the intertest reliability thus appears fairly close to the test245 retest reliability, which again means that we cannot expect to achieve a much higher intertest reliability than we 246 observed in this study. Nevertheless, although the ZNA-Q may capture slightly different information from the SB 
and DB components of the ZNA-2, the clear developmental trends observed in all tasks incorporated in the ZNA-

248 Q suggest that this test is applicable as a pre-screening tool for gross motor performance in preschool children to 249 assess the current developmental status of the child in clinical practice.

250 The ZNA-Q was established with a cohort of typically developing children. Because this cohort included few 251 children with gross motor problems or delay, future research should focus on children with motor difficulties and 252 how reliably the ZNA-Q is able to identify them.

\section{Acknowledgments}

255 We gratefully acknowledge the support of the presidents' conference of the public schools in Zurich and the 256 educators and teachers of the child care centres for their help with recruitment. This study was supported by the 257 Swiss National Science Foundation, grant no. 32003B_153273.

\section{Authors' contributions}

260 THK: Performed data collection, coded and analysed the corresponding data and wrote the manuscript

261 AZ: Performed statistical analysis and modelling

262 EK: Performed data collection and coded the corresponding data

263 JC: Performed data collection and corrected the manuscript

264 VR: Assisted in statistical data analysis

265 BR: Contributed to the draft and gave advice on the assessment tools

266 OGJ: Corrected final draft of the manuscript

267 All authors reviewed and edited the manuscript

\section{$269 \quad$ Funding}

270 This study was supported by the Swiss National Science Foundation, grant no. 32003B_153273, the Largo and 271 Giedion Risch Foundation.

\section{Compliance with ethical standards}

274 Conflict of interest The authors declare that they have no conflict of interest

276 Informed consent Informed consent was obtained from all individual participants included in the study. Parents 277 provided written informed consent for their participating child and children consented orally. 
280 1. Anderson, P. (2002). Assessment and development of executive function (EF) during

2. Barnett, L., Hinkley, T., Okely, A. D., \& Salmon, J. (2013). Child, family and environmental correlates of children's motor skill proficiency. Journal of Science and Medicine in Sport, 16(4), 332-336. doi:1 .1016/j.jsams.2012.08.011

3. Barnett, L. M., Telford, R. M., Strugnell, C., Rudd, J., Olive, L. S., \& Telford, R. D. (2018). Impact of cultural background on fundamental movement skill and its correlates. Journal of Sports Sciences, 1-8. doi:1 .1080/02640414.2018.1508399

4. Bax, M., \& Gillberg, C. (2009). Development: normal/delayed/disordered. In J. Aicardi (Ed.), Diseases of the nervous system in childhood (3rd ed., pp. 891-901). London: Mac Keith Press

5. Bayley, N. (2006). Bayley Scales of Infant and Toddler Development (3nd ed.). San Antonio: Pearson

6. Bruininks, R. H., \& Bruininks, B. D. (2005). Bruininks-Oseretsky Test of Motor Proficiency - Second edition (BOT-2). Minneapolis, USA: Pearson

7. Burton, A. W., \& Miller, D. A. (1998). Movement skill assessment. Leeds, United Kingdom: Human Kinetics

8. Fisher, R. A. (1936). Statistical methods for research workers. 6th edition, revised. Edinburgh: Oliver and Boyd

9. Folio, M., \& Fewell, R. (2000). Peabody Developmental Motor Scales-2. Austin, USA: PRO-ED

1. Henderson, S. E., Sugden, D. A., Barnett, A. L., Petermann, F., Bös, K., \& Kastner, J. (2007). Movement Assessment Battery for Children - Second Edition (Movement ABC2) - Deutschsprachige Adaptation. London, UK: Harcourt Assessment

11. Jonsson, E., Seiger, A., \& Hirschfeld, H. (2005). Postural steadiness and weight distribution during tandem stance in healthy young and elderly adults. Clinical Biomechanics (Bristol, Avon), 20(2), 202-208. doi:1 .1016/j.clinbiomech.2004.09.008

12. Kakebeeke, T. H., Caflisch, J., Chaouch, A., Rousson, V., Largo, R. H., \& Jenni, O. G. (2013). Neuromotor development in children. Part 3: motor performance in 3- to 5-yearolds. Developmental Medicine and Child Neurology, 55(3), 248-256. doi:1 .1111/dmcn. 12034

13. Kakebeeke, T. H., Knaier, E., Chaouch, A., Caflisch, J., Rousson, V., Largo, R. H., \& Jenni, O. G. (2018). Neuromotor development in children. Part 4: new norms from 3 to 18 years. Developmental Medicine and Child Neurology. doi:1 .1111/dmcn.13793

14. Kakebeeke, T. H., Locatelli, I., Rousson, V., Caflisch, J., \& Jenni, O. G. (2012). Improvement in gross motor performance between 3 and 5 years of age. Perceptual and Motor Skills, 114(3), 795-806. doi:1 .2466/1 .13.25.PMS.114.3.795-806

15. Kiphard, E. J., \& Schilling, F. (2017). KTK - Körperkoordinationstest für Kinder Bern: Hogrefe Verlag

16. Largo, R. H., Caflisch, J. A., Hug, F., Muggli, K., Molnar, A. A., \& Molinari, L. (2001). Neuromotor development from 5 to 18 years. Part 2: associated movements. Developmental Medicine and Child Neurology, 43(7), 444-453.

17. Largo, R. H., Caflisch, J. A., Hug, F., Muggli, K., Molnar, A. A., Molinari, L., Sheehy, A., \& Gasser, S. T. (2001). Neuromotor development from 5 to 18 years. Part 1: timed performance. Developmental Medicine and Child Neurology, 43(7), 436-443.

18. Largo, R. H., Rousson, V., Caflisch, J. A., \& Jenni, O. G. (2007). Zurich Neuromotor Assessment. Zurich, Switzerland: AWE Verlag 
19. Logan, S. W., Robinson, L. E., Wilson, A. E., \& Lucas, W. A. (2012). Getting the fundamentals of movement: a meta-analysis of the effectiveness of motor skill interventions in children. Child: Care, Health and Development, 38(3), 305-315. doi:1 $.1111 / \mathrm{j} .1365-2214.2011 .01307 . x$

2. Morris, A. M., Williams, J. M., Atwater, A. E., \& Wilmore, J. H. (1982). Age and SexDifferences in Motor-Performance of 3 through 6 Year Old Children. Research Quarterly for Exercise and Sport, 53(3), 214-221. doi:Doi 1 $.1080 / 02701367.1982 .10609342$

21. Piper, M. C., \& Darrah, J. (1994). Motor Assessment of the Developing Infant: Elsevier

22. Rudd, J., Butson, M. L., Barnett, L., Farrow, D., Berry, J., Borkoles, E., \& Polman, R. (2016). A holistic measurement model of movement competency in children. Journal of Sports Sciences, 34(5), 477-485. doi:1 .1080/02640414.2015.1061202

23. Shrout, P. E., \& Fleiss, J. L. (1979). Intraclass correlations: uses in assessing rater reliability. Psychological Bulletin, 86(2), 420-428.

24. Ulrich, D. A. (2000). Test of Gross Motor Development, 2nd Ed. Austin, USA: Pro-ED. Inc.

25. Utesch, T., Bardid, F., Huyben, F., Strauss, B., Tietjens, M., De Martelaer, K., Seghers, J., \& Lenoir, M. (2016). Using Rasch modeling to investigate the construct of motor competence in early childhood. Psychology of Sport and Exercise, 24, 179-187. doi:1 .1016/j.psychsport.2016.03.001

26. Wei, G., \& Tanner, M. (1990). A Monte Carlo Implementation of the EM Algorithm and the Poor Man's Data Augmentation Algorithms. J Am Stat Assoc, 85(411), 699704.

27. Zimmer, R., \& Volkamer, M. (1987). Motoriktest für vier- bis sechsjährige Kinder [Motor Test for four- to six-year-olds]. Weinheim: Beltz Test 
354 Table I: Items measured on ordinal scales of the Zurich Neuromotor Assessment; D = dominant leg; ND = 355 nondominant leg

356

\begin{tabular}{lll}
\hline Components & Tasks & 3-6 years \\
\hline Static balance & Standing on one leg (D/ND) & Time in Scales (0 to 4) \\
& Tandem stance (eyes open) & Time in Scales (0 to 4) \\
Dynamic balance & Hopping on one leg (D/ND) & Count in Scales (0 to 4) \\
& Walking on a straight line forward & Scale (0 to 4) \\
& Jumping sideways & Scale (0 to 4) \\
\hline
\end{tabular}

357 
358 Table II: Test-retest reliabilities (rank correlation coefficients) for single tasks and components of the ZNA-Q

359 and rank correlations between components of the ZNA-Q and ZNA-2, with 95\% confidence intervals (brackets).

\begin{tabular}{lcc}
\hline & Test-retest reliability & Rank correlations \\
Static balance: & $(\mathrm{N}=37)$ & with ZNA-2 \\
Standing on one leg (D/ND) & $.39[.08 ; .64]$ & \\
Tandem stance & $.17[-.17 ; .47]$ \\
Dynamic balance: & $.41[.10 ; .65]$ & \\
Hopping on one leg (D/ND) & $.39[-.08 ; .64]$ & \\
Walking forward on a straight line & $.43[.12 ; .66]$ & $.61[.24 ; .75]$ \\
Jumping sideways & $.70[.48 ; .84]$ \\
\hline Components: & $.41[.09 ; .65]$ & $.48[.03 ; .68]$ \\
Static Balance & $.67[.44 ; .82]$ & $.20 ; .75]$ \\
Dynamic Balance & & \\
Total composite score & & \\
\hline
\end{tabular}


361 Figure 1. Developmental trend of the five gross motor skills tasks of the ZNA-Q. Top row:

362 expected ordinal score. Bottom row: cumulative probabilities to obtain a score below or equal 363 to $k$, with $k \in\{0,1,2,3\})$.

364 
Standing on one leg

Age: $p<.001 ;$ Sex: $p=.002$
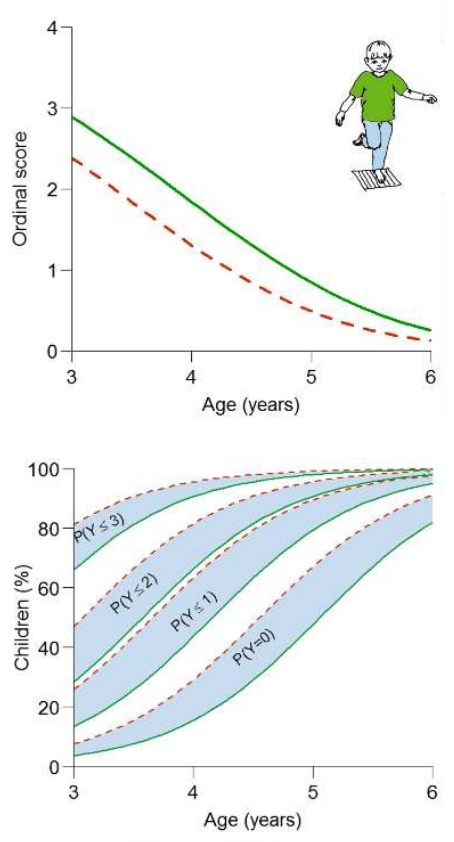

- Boys …. Girls

Score 0: can stand on both legs more than 5 seconds;

Score 1: can stand on one leg more than 5 seconds;

Score 2: can stand on both legs between 2 and 5 seconds;

Score 3: can stand on one leg between 2 and 5 seconds;

Score 4: cannot stand on either leg more than 2 seconds.
Tandem stance Age: $p<.001 ;$ Sex: $p=.131$
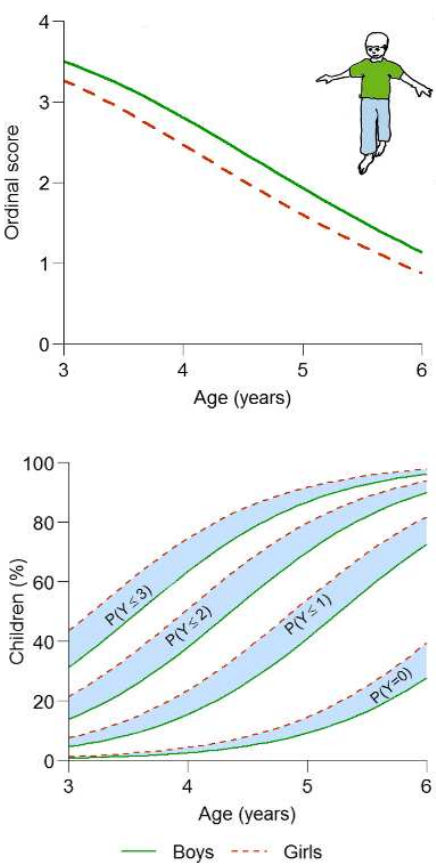

Score 0: can stand on one foot ahead of the other for 30 seconds or more;

core 1 : can stand on one foot ahead of the other between 10 and 30 seconds;

Score 2: can stand on one foot ahead of the other between 5 and 10 seconds; Score 3: can stand on one foot ahead of the other between 2 and 5 seconds: Score 4: cannot stand on one foot ahead
of the other for more than 2 sec.
Hopping on one leg

Age: $p<.001 ;$ Sex: $p=.107$
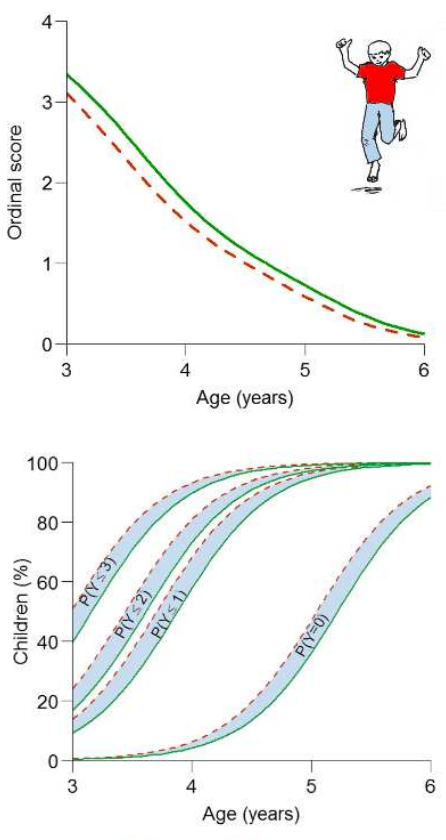

— Boys …. Girls

Score 0: can hop on both legs more than seven times;

Score 1: can hop on only one leg more than 3 times;

Score 2: can hop on both legs from one to 3 times;

Score 3: can hop on only one leg from one to 3 times;

Score 4: cannot hop on either leg.
Walking on a straight line

Age: $p<.001$; Sex: $p=.048$
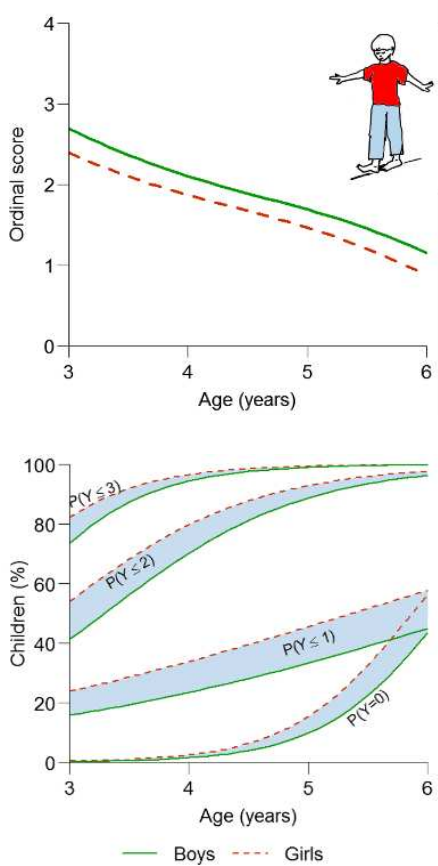

Score 0: perfect performance, heel touching toes;

core 1: distance between the two feet, feet straight;

Score 2: feet not straight and/or misses the line one to 3 times:

Score 3: feet perpendicular and/or does not touch the line more than 3

Score 4: fails to walk on a straight line.
Jumping sideways

Age: $p<.001 ;$ Sex: $p=.305$
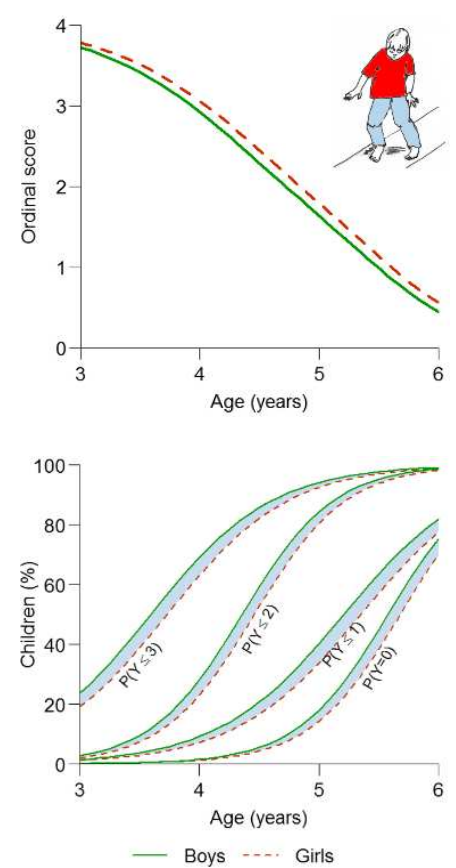

Score 0: perfect performance, very smooth jumping;

Score 1: fluently, but feet not parallel to rope $\left(<45^{\circ}\right)$;

Score 2: side to side, non-fluent, feet parallel to rope or $0<10^{\circ}$;

Score 3: side to side, non-fluent, feet not parallel to rope $\left(<45^{\circ}\right)$;

Score 4: jumping about but not in relation to the line. 